\section{Nuevas terapias orales de acción directa para tratamiento de virus de hepatitis C (VHC)}

\author{
JOSÉ IGNACIO VARGAS ${ }^{1}$, JUAN PABLO ARAB ${ }^{1}$, \\ HUGO MONRROY ${ }^{1}$, PILAR LABBÉ ${ }^{1}$, VALESKA SARMIENTO ${ }^{2}$, \\ FELIPE FUSTER ${ }^{2}$, FRANCISCO BARRERA ${ }^{1}$, CARLOS BENITEZ ${ }^{1}$, \\ MARCO ARRESE ${ }^{1}$, FRANCISCO FUSTER ${ }^{2,3}$, ALEJANDRO SOZA ${ }^{1}$
}

\section{Direct antivirals for the treatment of chronic hepatitis $C$ virus infection. Experience in 106 patients}

Background: The availability of direct-acting antivirals (DAA) for the treatment of chronic hepatitis $C$ virus (HCV) infection is just starting to expand in Chile. Aim: To report the initial experience of patients treated with $D A A$ and their evolution after treatment. Material and Methods: Prospective cohort study, from June 2013 to August 2016 of patients treated with DAA for $\mathrm{HCV}$ in three clinical centers. The presence of cirrhosis, clinical and laboratory features; adverse events (AE) and post-treatment changes in liver function were evaluated. Sustained viral response at 12 weeks post-treatment (SVR12) was determined. Results: One hundred six patients aged $58 \pm 13$ years, $54 \%$ males, were included. HCV genotype $1 \mathrm{~b}$ was present in $88 \%$ and $47 \%$ had cirrhosis. Treatment regimens were asunaprevir + daclatasvir (DCV) in 17\% of patients, paritaprevir / ritonavir / ombitasvir + dasabuvir in 33\%, sofosbuvir (SOF) + DCV in 19\%, and SOF + ledipasvir in 30\%. Twenty five percent of patients used generic drugs. SVR12 was $92.1 \%$, with no differences between generic and brand-name drugs. Serious AE were recorded in $22 \%$ of patients, being more common in those with cirrhosis ( $34 \%$ vs $11.5 \%, p<0.01)$. At 12 weeks post-treatment follow-up, there was a decrease in aminotransferase values $(p<0.01)$, improvement in Child-Pugh score (5.9vs. 5.5, $p=0.03$ ) and decreased presence of ascites ( $p=0.02)$. Conclusions: In our setting, DAA for HCV was highly effective and safe in non-cirrhotic patients. Hepatic function and inflammation improved at 12 weeks of follow-up. AE were common in patients with cirrhosis, suggesting that these patients should be treated by experienced teams. Generic drugs had similar effectiveness compared to originals.

(Rev Med Chile 2017; 145: 1235-1242)

Key words: Antiviral Agents; Chile; Drugs, Generic; Fibrosis; Hepatitis C; Latin America).

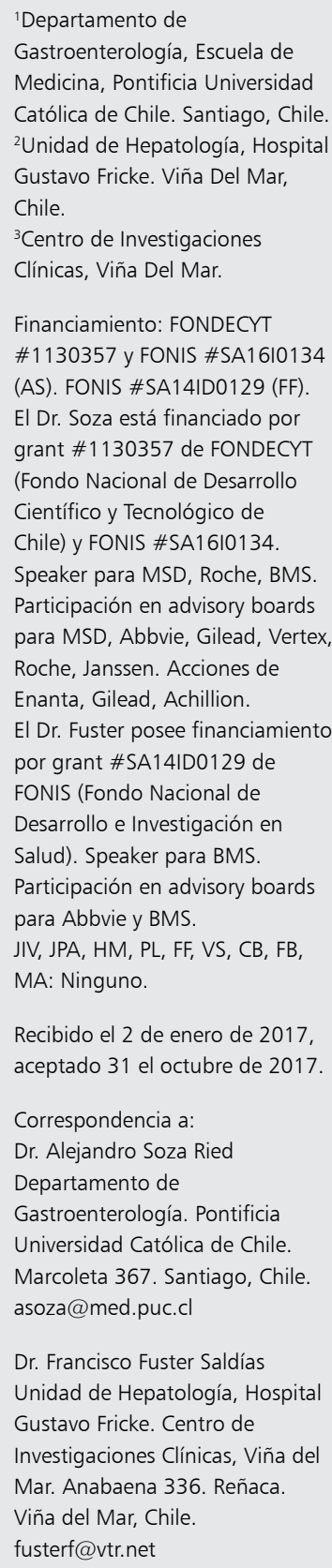

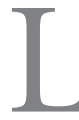
a infección crónica por virus de la hepatitis $\mathrm{C}$ (VHC) es una de las principales causas de cirrosis y carcinoma hepatocelular (CHC) en el mundo, constituyendo uno de los principales motivos de trasplante hepático ${ }^{1}$. Actualmente, se estima que existen cerca de 184 millones de per- sonas infectadas crónicamente con el VHC, unas 50.000 en Chile, y que la mayoría de ellas no ha sido diagnosticada ni tratada ${ }^{2}$.

Durante las últimas dos décadas se han logrado avances significativos en el manejo de los pacientes con infección crónica por el VHC gracias al mayor 
conocimiento de su fisiopatología, al desarrollo de nuevas técnicas diagnósticas y mejores alternativas terapéuticas ${ }^{3}$.

El objetivo principal del tratamiento es curar la infección, es decir, lograr una respuesta viral sostenida (RVS), definida como ARN indetectable de VHC a las 12-24 semanas después de haber terminado el tratamiento. Esto se correlaciona con normalización de pruebas de laboratorio hepático y considerable mejoría o desaparición de necroinflamación y fibrosis en la biopsia hepática ${ }^{4}$. Además, el riesgo de complicaciones derivadas de la cirrosis, como hipertensión portal, disminuyen con el tratamiento, sobre todo en aquellos pacientes que son tratados en etapas precoces de la enfermedad. Más aún, el riesgo de $\mathrm{CHC}$ también disminuye significativamente, aunque no se elimina completamente $e^{5,6}$.

La reciente disponibilidad de los denominados antivirales de acción directa $(\mathrm{AAD})$ ha revolucionado el tratamiento de la infección por el VHC, con altas tasas de curación, de simple administración y con pocos efectos adversos ${ }^{7}$. Esto permite pronosticar que los AAD se empezarán a utilizar de forma masiva, con un aumento del número de pacientes curados durante los próximos 15-20 años $^{8}$. En los países desarrollados, estas drogas se encuentran actualmente ampliamente utilizadas y validadas, con guías internacionales recomendando su uso en distintos esquemas ${ }^{4,9,10}$. En nuestro país, la guía clínica ministerial de manejo de infección por VHC, actualizada el año 2015, incorpora las nuevas terapias con antivirales de acción directa como primera línea con esquemas ajustados al genotipo, presencia de cirrosis y comorbilidades ${ }^{11}$. Sin embargo, pese a estar estos tratamientos disponibles y recomendados en nuestro país, el acceso aún es muy limitado. En el presente artículo, nos propusimos describir una experiencia inicial en Chile con el uso de antivirales de acción directa contra VHC y sus resultados en respuesta virológica, aparición de complicaciones, efecto en laboratorio y función hepática en el seguimiento temprano, posterior al término del tratamiento.

\section{Material y Métodos}

\section{Diseño del estudio}

La experiencia con uso de AAD comenzó en junio de 2013 en nuestro país, donde los primeros pacientes acceden a programas de uso compasivo de estos fármacos; posteriormente se fueron configurando otras formas de acceso, incluyendo reclutamiento dentro de protocolos clínicos, importación directa de fármacos originales y genéricos, programas de auxilio extraordinario del Ministerio de Salud. Lo anterior además de la compra directa en nuestro país, cuando estuvieron disponibles en el mercado local. Desde el inicio, se ha realizado un seguimiento y registro prospectivo de todos los pacientes tratados con AAD en los 3 centros participantes de este estudio (Hospital Clínico UC-CHRISTUS, Hospital Gustavo Fricke y Clínica Reñaca de Viña del Mar), configurando una cohorte prospectiva desde junio 2013, y realizando el análisis de datos recopilados en mayo 2017 para esta publicación. Las características basales de los pacientes fueron registradas considerando edad, género, comorbilidades, fármacos en uso, carga viral VHC, genotipo, tratamientos previos, función hepática basal y post tratamiento, función renal, parámetros hematológicos, presencia de cirrosis (definida por características clínicas, F4 en fibroscan y/o biopsia) y sus complicaciones, scores de Child-Pugh y MELD (Model for End Stage Liver Disease) basal y post tratamiento, tipo de AAD utilizados, duración del tratamiento, uso de ribavirina concomitante, eventos adversos al tratamiento, necesidad de suspensión de tratamiento, carga viral VHC a las 4, 12 y 24 semanas (si correspondió) y 12 semanas posterior al término del tratamiento (respuesta viral sostenida 12 semanas: RVS12).

\section{Análisis estadístico}

Se utilizó estadística descriptiva para evaluar características de la muestra mediante promedios, desviaciones estándar y proporciones. Se determinó el porcentaje de respuesta a AAD en forma global y por cada esquema de tratamiento basado en RVS12. Comparación de tasas de respuesta con distintos esquemas de tratamiento. Se realizó análisis inferencial por medio de $t$ de student, ANOVA, $\chi^{2}$, test de exacto de Fisher y test de McNemar para proporciones pareadas según correspondió, para cambios en variables de laboratorio, función hepática y renal, presencia de complicaciones de cirrosis previo, al finalizar tratamiento y 12 semanas posterior al término del tratamiento. Se realizó análisis de RVS por intención de tratar y por protocolo (excluyendo 
pacientes que se perdieron en seguimiento, pero incluyendo sujetos que suspendieron la terapia por eventos adversos).

\section{Resultados}

Un total de 106 pacientes fueron incluidos en el protocolo de seguimiento. De ellos, 5 se perdieron en seguimiento, siendo incluidos en análisis de respuesta por intención de tratamiento, quedando 101 pacientes con datos completos para análisis de respuesta por protocolo (Figura 1). La edad promedio de pacientes incluidos fue de 57,8 \pm 13 años, con $53,8 \%$ de género masculino. El genotipo $1 \mathrm{~b}$ fue predominante $(87,7 \%)$, coincidente con datos previos sobre genotipo VHC en nuestro país $^{12}$. Un $47,2 \%$ de pacientes tenían cirrosis al momento de inicio del tratamiento, la mayoría en categoría Child-Pugh A (70\%). El 70,8\% de ellos nunca había recibido tratamiento contra VHC, y $29,2 \%$ había tenido falla a algún esquema de tratamiento previo basado en interferón-pegilado más ribavirina. Los esquemas de tratamiento utilizados fueron variados, y en contextos diversos: programas de uso compasivo, protocolos clínicos, financiados directamente por el paciente, algunos con cobertura parcial por las aseguradoras, y otros en programa de Auxilio Extraordinario piloto del Ministerio de Salud. Un 25\% de los pacientes uti-

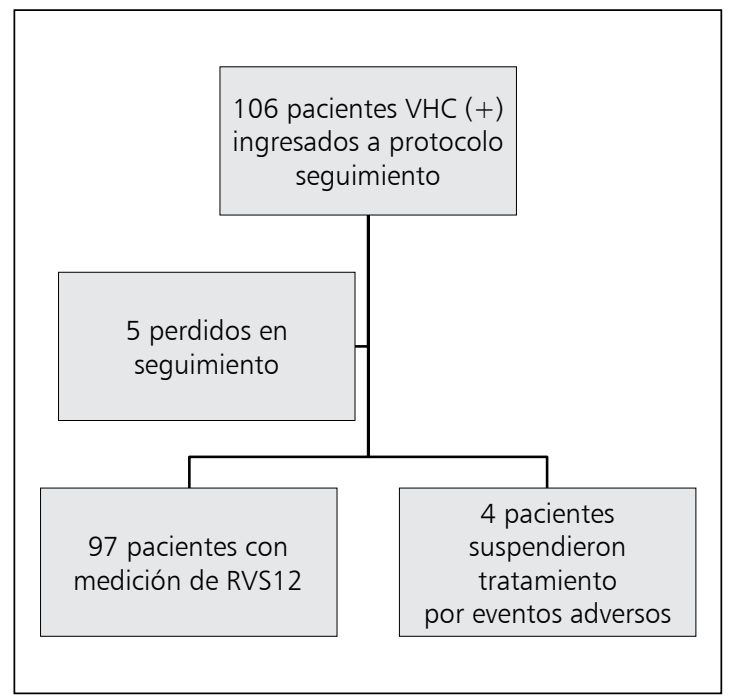

Figura 1. Flujograma de reclutamiento y seguimiento de pacientes en el estudio. lizó fármacos genéricos importados por iniciativa propia, en mayor parte desde la India. El 37,7\% de pacientes utilizó esquemas de AAD asociado a ribavirina, principalmente en cirróticos. Las características generales basales de pacientes incluidos en el estudio se detallan en la Tabla 1.

Tabla 1. Características generales de pacientes incluidos

\begin{tabular}{|c|c|}
\hline Variable & $\begin{array}{c}\text { Valor } \\
\text { (promedio } \pm \mathrm{DE} / \%)\end{array}$ \\
\hline $\mathrm{n}$ & 106 \\
\hline Edad (años) & $57,8( \pm 13,03)$ \\
\hline Género masculino (\%) & $53,8(57 / 106)$ \\
\hline $\begin{array}{l}\text { Genotipo VHC (\%) } \\
-1 b \\
-1 a \\
-2 \\
-3 \\
-4\end{array}$ & $\begin{array}{r}87,7(93 / 106) \\
4,71(5 / 106) \\
0,94(1 / 106) \\
3,77(4 / 106) \\
2,83(3 / 106)\end{array}$ \\
\hline $\begin{array}{l}\text { Carga viral VHC basal } \\
\text { (promedio copias/ml) }\end{array}$ & $\begin{array}{c}4.080 .320 \\
\left( \pm 5,4 \times 10^{6}\right)\end{array}$ \\
\hline $\begin{array}{l}\text { Tratamiento previo fallido contra } \\
\text { VHC en base a PEG-IFN (\%) }\end{array}$ & $29,3(31 / 106)$ \\
\hline $\begin{array}{l}\text { Esquema tratamiento (\%): } \\
\text { - ASV-DCV } \\
\text { - 3D } \\
\text { - SOF-DCV } \\
\text { - SOF-LDV } \\
\text { - SOF-SIM }\end{array}$ & $\begin{array}{r}16,9(18 / 106) \\
32,0(35 / 106) \\
18,9(20 / 106) \\
30,2(32 / 106) \\
0,94(1 / 106)\end{array}$ \\
\hline $\begin{array}{l}\text { Uso de ribavirina asociado a AAD (\%) } \\
\text { - Cirróticos (\%) }\end{array}$ & $\begin{array}{l}37,7(40 / 106) \\
58,0(29 / 50)\end{array}$ \\
\hline Fármacos genéricos (\%) & $25,0(26 / 106)$ \\
\hline $\begin{array}{l}\text { Cirrosis (\% total) } \\
- \text { - A } \\
- \text { B } \\
\text { - C }\end{array}$ & $\begin{array}{c}47,2(50 / 106) \\
70,0(35 / 50) \\
22,0(11 / 50) \\
6,0(3 / 50)\end{array}$ \\
\hline $\begin{array}{l}\text { MELD basal en pacientes con } \\
\text { cirrosis (promedio) }\end{array}$ & $7,87( \pm 4,03)$ \\
\hline $\begin{array}{l}\text { Várices esofágicas (\%) en } \\
\text { cirróticos }\end{array}$ & $68,0(34 / 50)$ \\
\hline Ascitis (\%)en cirróticos & $28,0(14 / 106)$ \\
\hline $\begin{array}{l}\text { Encefalopatía hepática (\%) } \\
\text { en cirróticos }\end{array}$ & $10,0(5 / 106)$ \\
\hline $\begin{array}{l}\text { Recuento de plaquetas } \\
\left(\mathrm{cel} / \mathrm{mm}^{3}\right) \times 10^{3}\end{array}$ & $168,5( \pm 102,7)$ \\
\hline Albúmina (g/dl) & $4,0( \pm 0,54)$ \\
\hline
\end{tabular}




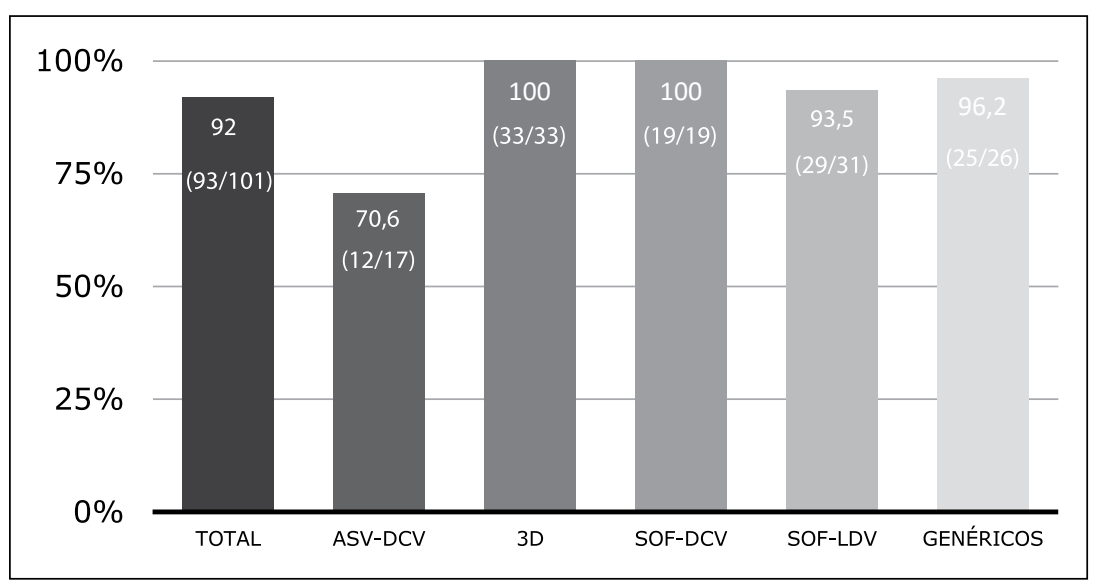

Figura 2. Porcentaje de RVS12 a antivirales de acción directa en análisis por intención de tratamiento, y diferencias según esquema de antivirales de acción directa usados. Respuesta a antivirales de acción directa. ASVDCV: Asunaprevir-Daclatasvir, 3D: Paritaprevir-ritonavir, Ombitasvir, Dasabuvir, SOF-DCV: Sofosbuvir-Daclastavir, SOFLDV: Sofosbuvir-Ledipasvir., Genéricos (SOF-DCV, SOF-LDV).
Tabla 2. Tabla comparativa entre RVS12 en según presencia de cirrosis y tratamiento previo

\begin{tabular}{|lcc|}
\hline Variable & RVS12 (\%) & p value \\
\hline Cirróticos & $85,1 \%(40 / 47)$ & 0,024 \\
No cirróticos & $98,1 \%(53 / 54)$ & \\
Tratamiento previo & $86,2 \%(25 / 29)$ & 0,222 \\
Naive & $94,4 \%(68 / 72)$ & 2 \\
\hline
\end{tabular}

En análisis por intención de tratamiento, la respuesta viral sostenida (RVS12) en el total de pacientes incluidos en el seguimiento fue de $87,8 \%$ (93/106) (IC 95\% 80-93,3). En análisis por protocolo, excluyendo a pacientes que fueron perdidos en seguimiento, la respuesta fue de 92,1\% (93/101) (IC95\% 85,7-96,7). La RVS12 para ASV-DCV (Asunaprevir-Daclatasvir) fue 70,6\% (12/17), $100 \%$ para SOF-DCL (Sofosbuvir-Daclastavir, 19/19) y 3D (Paritaprevir-ritonavir, Ombitasvir, Dasabuvir; 33/33), 93,5\% (29/31) para SOF-LDV (Sofosbuvir-Ledipasvir) y sólo 1 paciente usó SOFSIM (Sofosbuvir-Simeprevir). El tratamiento con fármacos genéricos (SOF-LDV y SOF-DCV) tuvo 96,2\% (25/26) de eficacia (Figura 2). No hubo diferencias significativas en RVS12 entre los diferentes esquemas utilizados $(\mathrm{p}=0,154)$.

La RVS12 fue significativamente mayor en pacientes no cirróticos comparados a pacientes cirróticos, quienes tuvieron más falla o abandono de tratamiento $(98,1 \%$ [53/54] RVS12 en no cirróticos versus 85,1\% [40/47] RVS12 en cirróticos, $\mathrm{p}=0,024)$. No hubo diferencias significativas en RVS12 según el estatus previo de tratamiento: pacientes que ya habían sido previamente tratados con régimen en base a interferón-pegilado tuvie-
Tabla 3. Eventos adversos asociados al tratamiento con antivirales de acción directa para VHC

\begin{tabular}{|lc|}
\hline 1. Eventos adversos serios & $\mathbf{n = 2 0 / 8 7 ~ ( 2 3 \% ) ~}$ \\
Muerte: & 3 \\
- Descompensación hepática & 2 \\
- Sepsis foco biliar & 1 \\
Neoplásicos: & 6 \\
- HCC & 2 \\
- Mieloma múltiple & 1 \\
- Cáncer de próstata & 1 \\
- Cáncer de colon & 1 \\
- Colangiocarcinoma & 1 \\
Hematológicos: & 7 \\
- Anemia severa sintomática & 6 \\
- Neutropenia transitoria & 1 \\
Hepáticos: & 2 \\
- Ascitis refractaria & 2 \\
Otros: & 2 \\
- Episodio convulsivo & 1 \\
- Dermatitis & 1 \\
2. Eventos adversos leves & $\mathbf{2 3 / 8 7} \mathbf{( 2 6 , 4 \% )}$ \\
- Compromiso estado general & 7 \\
- Dolor abdominal & 3 \\
- Nauseas & 2 \\
- Diarrea & 2 \\
- Disestesias extremidades & 3 \\
- Lesiones cutáneas & 3 \\
- Cefalea & 2 \\
- Infección respiratoria baja & 1 \\
\hline
\end{tabular}

ron la misma respuesta que pacientes que eran tratados por primera vez (86,2\% [25/29] RVS12 en previamente tratados versus $94,4 \%$ [68/72] RVS12 en pacientes sin tratamiento previo, $\mathrm{p}=0,222$ ).

Durante el tratamiento, $22,2 \%$ de pacientes presentó algún evento adverso serio, lo que fue 
significativamente más frecuente en pacientes cirróticos $(34,0 \%$ en cirróticos versus $11,5 \%$ en no cirróticos, $\mathrm{p}=0,007)$. Los eventos adversos serios llevaron a suspender el tratamiento en 4 pacientes, 3 fallecieron al poco tiempo: 2 por descompensación hepática y 1 por sepsis de foco biliar, además, un paciente suspendió el tratamiento por diagnóstico de cáncer de colon; hubo progresión o diagnóstico de neoplasias en 6 pacientes, aumento de ascitis haciéndose refractaria a diuréticos en 2 pacientes; complicaciones hematológicas, fundamentalmente anemia, en 7 pacientes, todas asociadas al uso de ribavirina. Los eventos adversos serios fueron más frecuentes en cirróticos que presentaban ascitis al inicio del tratamiento (50\% versus $17,6 \%, p=0,013)$.

Se presentaron eventos adversos leves en $27,2 \%$ de los pacientes, sin diferencias en relación a presencia de cirrosis $(23,8 \%$ en cirróticos versus $30,0 \%$ en no cirróticos, $\mathrm{p}=0,506)$. La mayoría de efectos adversos leves fueron síntomas constitucionales asociados a la terapia. La incidencia de eventos adversos serios y leves durante el tratamiento se detallan en la Tabla 3.

Se controló la evolución de pruebas hepáticas inmediatamente posterior al término del tratamiento y 12 semanas después, cuyos resultados se muestran en la Figura 3.

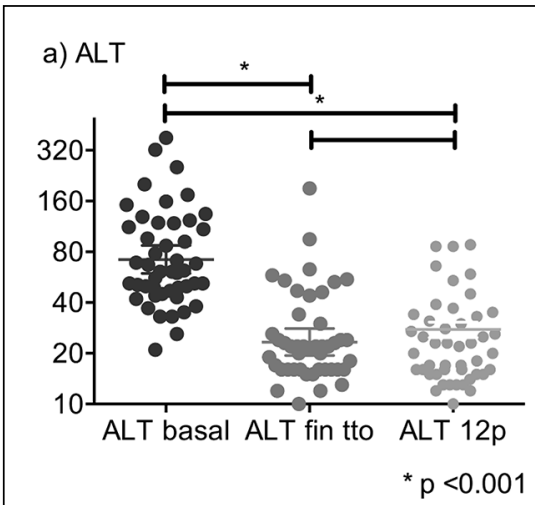

b) Bilirrubina total

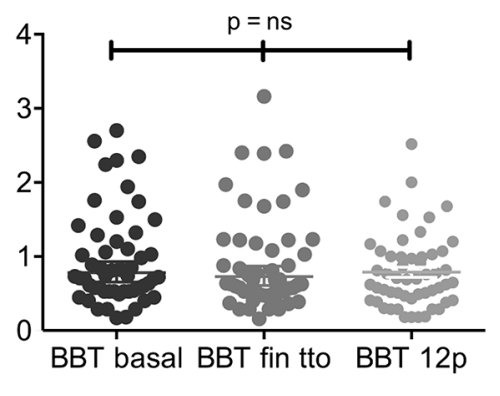

c) Albúmina

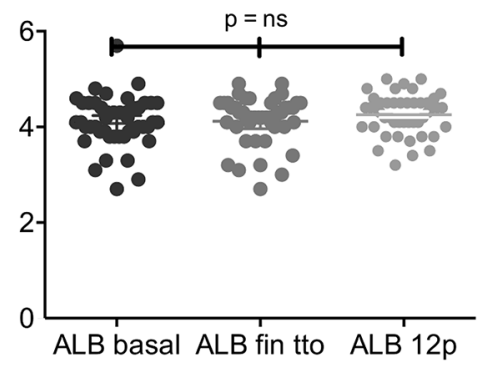

d) INR

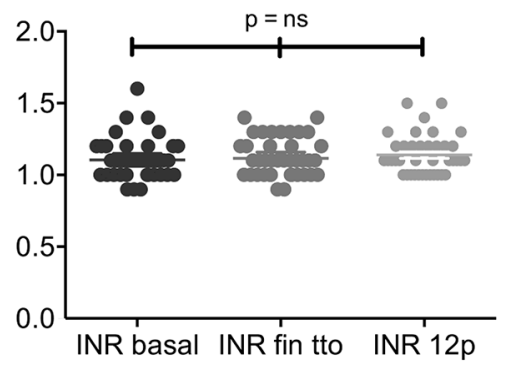

e) Creatinina

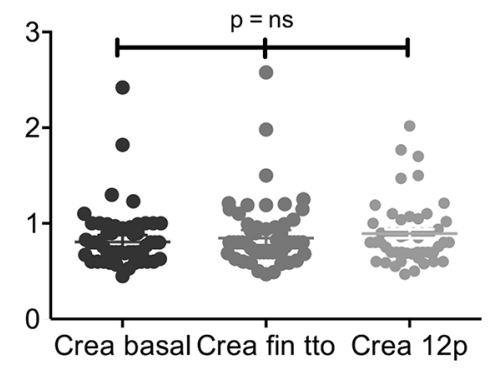

Figura 3. Evolución pruebas hepáticas inicio y término de tratamiento y control a las 12 semanas post tratamiento $(n=101)$. 

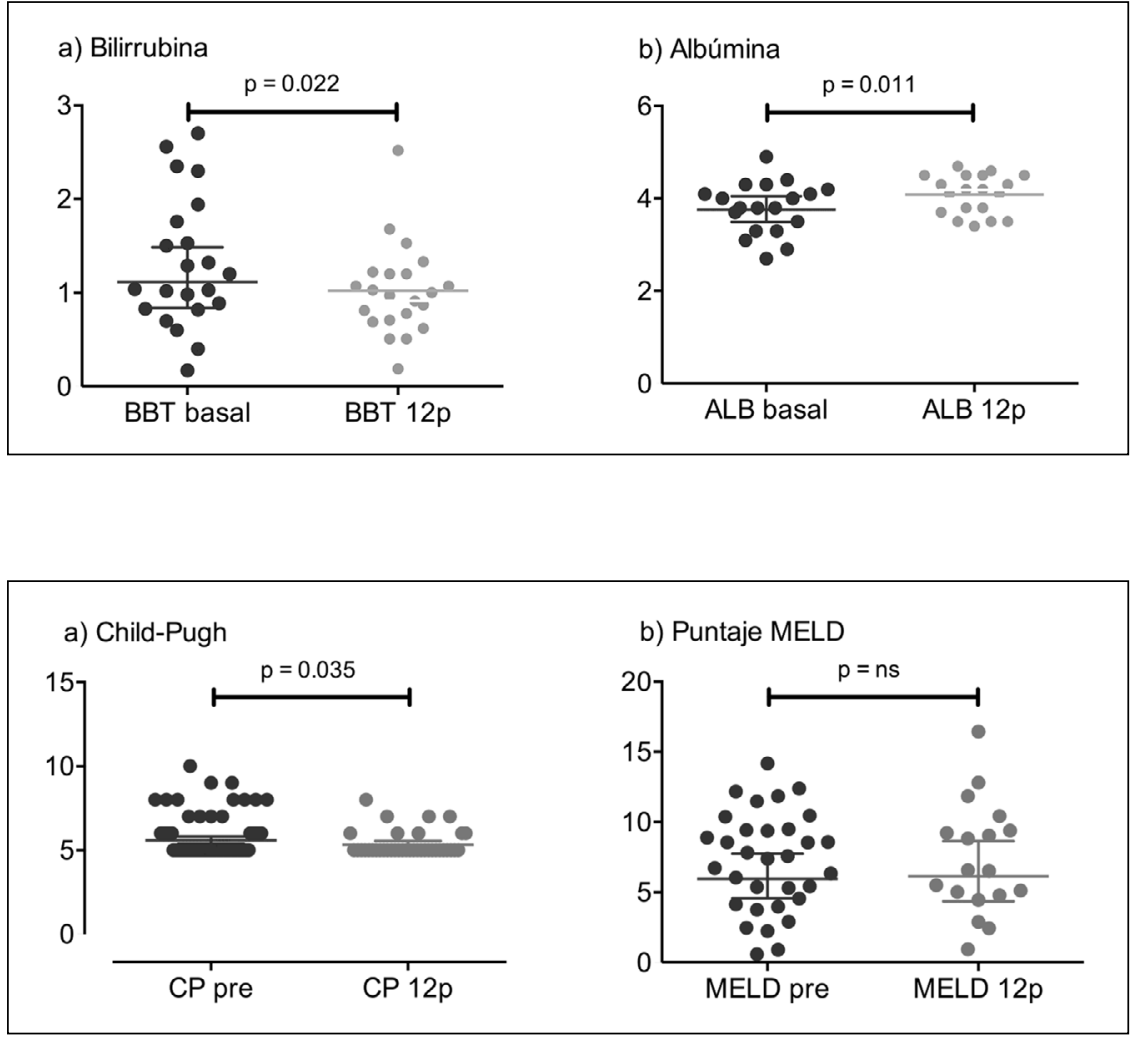

Figura 4. Cambios en Bilirrubina total y Albúmina en pacientes cirróticos que lograron RVS12 $(n=40)$.
Figura 5. Cambios en puntajes de Child-Pugh y MELD basales y 12 semanas post tratamiento $(n=40)$.
Hubo una disminución significativa en valores de alanino aminotransferasa (ALT/SGPT) al término del tratamiento, descenso que se mantuvo al control de las 12 semanas ( $\mathrm{p}<0,001)$. Al considerar la muestra completa, no hubo diferencias en valores de bilirrubina total, albúmina, INR o creatinina comparado a los niveles basales. Sin embargo, al analizar el subgrupo de pacientes cirróticos que lograron RVS12 (40 pacientes) se observó un significativo descenso en valores de Bilirrubina total a las 12 semanas posterior al fin del tratamiento comparado al basal $(1,32 \pm 0,7$ versus $1,02 \pm 0,5, p=0,022)$, además, de un aumento en niveles séricos de Albúmina (3,8 $\pm 0,5$ versus 4,09 $\pm 0,4, p=0,011$ ) (Figura 4).

El promedio de puntaje Child-Pugh fue significativamente menor en los pacientes cirróticos que completaron el tratamiento antiviral en el control a las 12 semanas post tratamiento $(5,9$ vs 5,5 , $p=0,035)$. No hubo diferencias en puntaje MELD, considerando que la mayoría de los pacientes se encontraban en categoría de Child-Pugh A con una buena función sintética hepática (Figura 5).

De los 6 pacientes cirróticos con encefalopatía hepática al inicio del tratamiento, 1 paciente suspendió el tratamiento por una nueva descompensación hepática falleciendo poco después, en 3 pacientes se mantuvo la encefalopatía 12 semanas post tratamiento y 2 pacientes tuvieron mejoría de encefalopatía post tratamiento.

La presencia de ascitis disminuyó significativamente posterior al tratamiento, teniendo al inicio una prevalencia de $14 \%$ comparado a $5 \%$ al control de las 12 semanas posterior al término del tratamiento $(\mathrm{p}=0,02)$.

\section{Discusión}

El presente estudio muestra los resultados de tratamientos con AAD en nuestro país, el cual se asocia a altas tasas de respuesta, concordantes con las publicadas tanto en estudios clínicos como en experiencias de vida real ${ }^{13}$. Tener una confirma- 
ción local de efectividad de terapias orales para infección crónica por $\mathrm{VHC}$ es relevante en el contexto de las decisiones que las autoridades de salud deben tomar en relación a ampliar el acceso de estos tratamientos con mecanismos específicos, como su incorporación al sistema de Garantías Explícitas de Salud (GES). Nuestros resultados muestran que la tasa de curación (RVS12) es de más de $90 \%$, lo que se compara muy favorablemente con tasas de respuesta que bordean $50 \%$ o menos con peginterferón y ribavirina. Estas altas tasas de respuesta se comprobaron independientemente de presencia de factores que pueden asociarse a menor respuesta, como cirrosis, edad avanzada, genotipo 1 y falla a tratamiento previo ${ }^{4}$.

La experiencia expuesta muestra no sólo altas tasas de respuesta, sino en general que el tratamiento es muy bien tolerado. A este respecto se debe destacar que hubo muy baja aparición de eventos adversos en el grupo de pacientes sin cirrosis, sin embargo, sí encontramos aparición de eventos adversos serios en el grupo de pacientes con cirrosis. Este grupo representaba $47 \%$ de los pacientes y es relevante, ya que la apertura del acceso a nuevas terapias probablemente priorice a pacientes con mayor fibrosis o con cirrosis constituida. Los eventos adversos serios se concentraron en este grupo, lo que sugiere que estos enfermos deben ser tratados en centros con experiencia en el manejo de pacientes con enfermedad hepática avanzada.

Merece mención especial el hecho de que 6 pacientes desarrollaron o tuvieron progresión de neoplasias durante el tratamiento o en el seguimiento posterior a terapia con AAD. Algunos reportes recientes sugieren que el tratamiento con AAD podría asociarse a un riesgo aumentado de recurrencia de hepatocarcinoma. Se conjetura que el mecanismo podría ser una disminución de la vigilancia inmunológica luego de la erradicación del virus ${ }^{14,15}$. Otro estudio con mayor número de pacientes, sin embargo, no mostró mayores tasas de recurrencia ${ }^{16}$ y un metaanálisis reciente concluye que el beneficio ya demostrado en sobrevida de la terapia basada en interferón está específicamente ligada a la consecución de $\mathrm{RVS}^{17}$, lo que sugiere que la terapia antiviral con AAD, por su alta probabilidad de lograr RVS, puede considerarse como una terapia adyuvante del $\mathrm{HCC}^{17}$. En nuestra experiencia, sólo 2 de los 6 casos de neoplasia fueron HCC, siendo interesante de destacar y que al menos debe dejar el mensaje de que debemos estar atentos al desarrollo de neoplasias en estos enfermos, sin que podamos establecer una relación causal.

El desarrollo de anemia fue uno de los eventos adversos más frecuentes y se asoció, como era esperado, al uso de ribavirina y a la presencia de cirrosis hepática. Un control frecuente del paciente en base a los síntomas y hemograma son la base de la prevención y manejo de anemia en este grupo de enfermos, que habitualmente fueron manejados en forma exitosa mediante reducciones de la dosis de ribavirina, sin tener que llegar a requerir transfusiones o uso de estimuladores como eritropoyetina, permitiendo completar el tratamiento.

Nos parece interesante recalcar que con seguimiento de sólo 12 semanas post-fin de tratamiento no sólo son evidentes mejorías en parámetros inflamatorios (ALT), sino que también se evidencian mejorías en función hepática, particularmente en pacientes con cirrosis, con mejorías en puntaje de Child-Pugh y en síntomas relevantes como ascitis y encefalopatía hepática. Estas mejorías debieran ir haciéndose más evidentes a medida que aumenta el tiempo de seguimiento de los pacientes, y representan el beneficio más claro de expandir el acceso a estas terapias.

En nuestro estudio, 25\% de pacientes incluidos utilizó fármacos genéricos para el tratamiento de infección crónica por VHC, con una alta tasa de respuesta virológica $(96,2 \%)$. Esto es coincidente con un estudio publicado recientemente que describe una alta tasa de respuesta con el uso de Sofosbuvir y Ledipasvir genéricos en pacientes cirróticos y no-cirróticos ${ }^{18}$. Según la experiencia local, el costo del tratamiento con fármacos genéricos podría ser hasta $90 \%$ menor que el uso de fármacos originales. Esto constituye una alternativa especialmente atractiva en países en vías de desarrollo, o cuando no existe cobertura de estos tratamientos antivirales por seguros de salud y deben ser costeados por los propios pacientes ${ }^{19}$.

Recomendaciones actuales ${ }^{4,10}$ establecen que todos los pacientes con infección crónica por VHC deben ser considerados para tratamiento, excepto aquellos con expectativa de vida limitada por causas extra-hepáticas. En caso de existir limitación de recursos para proveer tratamiento universal, entonces es más apropiado priorizar el tratamiento a aquellos con enfermedad más avanzada y con mayor riesgo de complicaciones. 
Dado que las guías ministeriales actuales de manejo de VHC en Chile incluyen como primera línea de tratamiento los nuevos $\mathrm{AAD}^{11}$, parece relevante contar con una experiencia local que confirme la efectividad de estas terapias y que, a la vez, permita poner atención a potenciales problemas y eventos adversos asociados a su uso. En nuestra opinión, el balance es claramente beneficioso, faltando solamente el paso de hacer que estas terapias estén más ampliamente disponibles mediante una expansión del acceso por la vía del GES.

\section{Referencias}

1. Mohd Hanafiah K, Groeger J, Flaxman AD, Wiersma ST. Global epidemiology of hepatitis $\mathrm{C}$ virus infection: new estimates of age-specific antibody to HCV seroprevalence. Hepatology 2013; 57 (4): 1333-42.

2. Messina JP, Humphreys I, Flaxman A, Brown A, Cooke GS, Pybus OG, et al. Global distribution and prevalence of hepatitis C virus genotypes. Hepatology 2015; 61 (1): 77-87.

3. Götte M, Feld JJ. Direct-acting antiviral agents for hepatitis C: structural and mechanistic insights. Nat Rev Gastroenterol Hepatol 2016; 13 (6): 338-51.

4. European Association for the Study of the Liver. Electronic address: easloffice@easloffice.eu. EASL recommendations on treatment of hepatitis C 2016. J Hepatol 2017; 66 (1): 153-94.

5. Bruno S, Di Marco V, Iavarone M, Roffi L, Crosignani A, Calvaruso V, et al. Survival of patients with $\mathrm{HCV}$ cirrhosis and sustained virologic response is similar to the general population. J Hepatol 2016; 64 (6): 1217-23.

6. van der Meer AJ, Veldt BJ, Feld JJ, Wedemeyer H, Dufour J-F, Lammert F, et al. Association between sustained virological response and all-cause mortality among patients with chronic hepatitis $\mathrm{C}$ and advanced hepatic fibrosis. JAMA 2012; 308 (24): 2584-93.

7. Majumdar A, Kitson MT, Roberts SK. Systematic review: current concepts and challenges for the direct-acting antiviral era in hepatitis $\mathrm{C}$ cirrhosis. Aliment Pharmacol Ther 2016; 43 (12): 1276-92.

8. Towards elimination of viral hepatitis by 2030 . The Lancet 2016; 388 (10042): 308.

9. Méndez-Sánchez N, Paraná R, Cheinquer H, Alves de Mattos A, Gadano A, Silva M, et al. Latin American
Association for the Study of the Liver recommendations on treatment of hepatitis C. Ann Hepatol. 2014 Aug;13 Suppl 2:s4-66.

10. Terrault NA, Bzowej NH, Chang K-M, Hwang JP, Jonas MM, Murad MH, et al. AASLD guidelines for treatment of chronic hepatitis B. Hepatology. 2016 Jan;63(1):26183.

11. Ministerio de Salud. Guía Clínica de Manejo de la Infección Crónica por Virus de la Hepatitis C (VHC). Santiago, Chile. Julio 2015.

12. Soza A, Arrese M, González R, Alvarez M, Pérez RM, Cortés $\mathrm{P}$, et al. Clinical and epidemiological features of 147 Chilean patients with chronic hepatitis C. Ann Hepatol 2004; 3 (4): 146-51.

13. Conti F, Brillanti S, Buonfiglioli F, Vukotic R, Morelli MC, Lalanne C, et al. Safety and efficacy of direct-acting antivirals for the treatment of chronic hepatitis $\mathrm{C}$ in a real-world population aged 65 years and older. J Viral Hepat 2017; 24 (6): 454-63.

14. Conti F, Buonfiglioli F, Scuteri A, Crespi C, Bolondi L, Caraceni P, et al. Early occurrence and recurrence of hepatocellular carcinoma in HCV-related cirrhosis treated with direct-acting antivirals. J Hepatol 2016; 65 (4): 727-33.

15. Reig M, Mariño Z, Perelló C, Iñarrairaegui M, Ribeiro A, Lens S, et al. Unexpected high rate of early tumor recurrence in patients with HCV-related HCC undergoing interferon-free therapy. J Hepatol 2016; 65 (4): 719-26.

16. ANRS collaborative study group on hepatocellular carcinoma (ANRS CO22 HEPATHER, CO12 CirVir and CO23 CUPILT cohorts). Electronic address: stanislas. pol@aphp.fr. Lack of evidence of an effect of direct-acting antivirals on the recurrence of hepatocellular carcinoma: Data from three ANRS cohorts. J Hepatol 2016; 65 (4): 734-40.

17. Manthravadi S, Paleti S, Pandya P. Impact of sustained viral response postcurative therapy of hepatitis C-related hepatocellular carcinoma: a systematic review and meta-analysis. Int J Cancer 2017; 140 (5): 1042-9.

18. Zeng Q-L, Xu G-H, Zhang J-Y, Li W, Zhang D-W, Li $Z-Q$, et al. Generic ledipasvir-sofosbuvir for patients with chronic hepatitis C: A real-life observational study. J Hepatol 2017; 66 (6): 1123-9.

19. Lim SG. HCV management in resource-constrained countries. Hepatol Int 2017; 11 (3): 245-54. 